\title{
Evaluation of Clonostachys rosea for Control of Plant-Parasitic Nematodes in Soil and in Roots of Carrot and Wheat
}

\author{
Mudassir Iqbal,† Mukesh Dubey, Kerstin McEwan, Uwe Menzel, Mikael Andersson Franko, Maria Viketoft, \\ Dan Funck Jensen, and Magnus Karlsson
}

First, second, fourth, seventh, and eighth authors: Department of Forest Mycology and Plant Pathology, Uppsala BioCenter, Swedish University of Agricultural Sciences, Box 7026, 75007 Uppsala, Sweden; third and fifth authors: Department of Energy and Technology, Swedish University of Agricultural Sciences, Box 7032, 75007 Uppsala, Sweden; and sixth author: Department of Ecology, Swedish University of Agricultural Sciences, Box 7044, 75007 Uppsala, Sweden.

Accepted for publication 8 September 2017.

ABSTRACT

\begin{abstract}
Biological control is a promising approach to reduce plant diseases caused by nematodes. We tested the effect of the fungus Clonostachys rosea strain IK726 inoculation on nematode community composition in a naturally nematode infested soil in a pot experiment, and the effect of $C$. rosea on plant health. The numbers of plant-parasitic nematode genera extracted from soil and plant roots decreased by 40 to $73 \%$ when C. rosea was applied, while genera of nonparasitic nematodes were not affected. Soil inoculation of $C$. rosea increased fresh shoot weight and shoot length of wheat plants by 20 and $24 \%$, respectively, while only shoot dry weight increased by $48 \%$ in carrots. Light microscopy of in vitro C. roseanematode interactions did not reveal evidence of direct parasitism.
\end{abstract}

However, culture filtrates of $C$. rosea growing in potato dextrose broth, malt extract broth and synthetic nutrient broth exhibited toxicity toward nematodes and immobilized 57,62, and $100 \%$ of the nematodes, respectively, within $48 \mathrm{~h}$. This study demonstrates that $C$. rosea can control plant-parasitic nematodes and thereby improve plant growth. The most likely mechanism responsible for the antagonism is antibiosis through production of nematicidal compounds, rather than direct parasitism.

Additional keywords: biological control, integrated pest management, plant growth parameters.
Nematodes belong to the phylum Nematoda (kingdom Animalia), and display an extensive range of feeding habits, including plant parasitic, bacterivores, fungivores, predators, and omnivores. There are 4,100 characterized species of plant-parasitic nematodes and there are three main divisions of plant parasitism; i.e., endoparasitic (Meloidogyne spp. and Heterodera spp.), ectoparasitic (Trichodorus spp. and Rotylenchus spp. etc.), and semiendoparasitic (Tylenchulus spp.) nematodes (Decraemer and Hunt 2013).

The sedentary endoparasitic nematodes are among the most destructive agricultural pests, attacking an extensive variety of crops (Williamson and Gleason 2003). Second-stage juveniles (J2) hatch from eggs in the soil and subsequently enter roots and start the infection (Milligan et al. 1998; Sharon et al. 2001). All life stages of migratory endoparasitic nematodes can be found inside host tissues (Moens and Perry 2009) and have great negative impact on crop production all over the world (Ganguly and Ganguly 2008). Plantparasitic nematodes are able to reduce both yield and quality of the crop. They are estimated to cause on average up to $14.6 \%$ crop production losses in agriculture and forestry worldwide, which is equal to 118 billion U.S. dollars annually, but sometimes leading to complete loss of marketable yield (Barker and Koenning 1998; Nicol et al. 2011). Chemical control has proven to effectively control plant-parasitic nematodes in many cases (Barker and Koenning 1998). However, because of their high cost, toxicity to livestock, plants, biodiversity (Beketov et al. 2013), development of resistance in nematode pathogen populations (Viglierchio and Brown 1989; Yamashita et al. 1986), and banned chemical soil disinfestation for nematode control in many countries, chemical control of nematode diseases is today limited. Furthermore, chemical control is not allowed in organic production,

†Corresponding author: M. Iqbal; E-mail: mudassir.iqbal@slu.se

(C) 2018 The American Phytopathological Society which emphasizes the need for development and implementation of alternative control strategies.

Certain soilborne microorganisms, including the nematodeantagonistic and mycoparasitic fungus Clonostachys rosea (Link: Fr.) Schroers, Samuels, Seifert \& W. Gams, comb. nov. (Schroers et al. 1999), are proposed as potential biological agents to control plant-parasitic nematodes (Dong et al. 2005; Zou et al. 2010). Certain Clonostachys strains can potentially affect a range of different plant-parasitic nematodes, through direct parasitism on several nematode life stages, including eggs and cysts (Zou et al. 2010). C. rosea is also reported to produce nematicidal compounds such as leptosins, chetoracin A, chaetocin, and gliocladines A, B, C, D, and E (Dong et al. 2005), and enzymes such as extracellular serine proteases (Zou et al. 2010) and chitinases (Gan et al. 2007; Tzelepis et al. 2015), which may be involved in the biocontrol effect against plant-parasitic nematodes. Some reports suggest that certain strains of $C$. rosea can interact with plant roots and trigger local and systemic resistance that suppress fungal diseases (Mouekouba et al. 2014; Roberti et al. 2008), while the effect of induced resistance against nematodes needs to be studied further. The genome sequence of $C$. rosea strain IK726 was recently determined (Karlsson et al. 2015) and shown to contain high numbers of genes predicted to be involved in the biosynthesis of natural products, including polyketide synthases and nonribosomal peptide synthetases, but relatively few chitinases (Tzelepis et al. 2015). This strain is an efficient biological control agent against several fungal and oomycete plant pathogens (Jensen et al. 2007), but its efficiency for nematode antagonism is not yet studied.

The present study was designed to test the effect of $C$. rosea strain IK726 on nematodes in a naturally nematode infested soil and on plant health, under controlled conditions. The used nematode community consisted of different trophic groups but was dominated by the genus Pratylenchus; therefore, carrot and wheat were chosen as plant hosts. We hypothesize that (i) C. rosea IK726 can antagonize and decrease 
nematode numbers in soil and roots of carrot and wheat, (ii) $C$. rosea IK726 antagonism is nonspecific, affecting all genera and nematode groups similarly, (iii) the activity of $C$. rosea IK726 in the soil improves growth of carrot and wheat, and (iv) C. rosea IK726 antagonism involve both direct parasitism of nematodes and production of secreted nematicidal metabolites and enzymes.

\section{MATERIALS AND METHODS}

Soil sampling. Soil containing a naturally diverse community of nematodes, including plant-parasitic genera, was collected in November 2015 from a wheat field in Boländerna, (coordinates: $59^{\circ} 50^{\prime} 09.3^{\prime \prime} \mathrm{N}, 17^{\circ} 41^{\prime} 52.5^{\prime \prime} \mathrm{E}$ ), close to Uppsala, Sweden. Soil was collected from four different places in the same field during fallow conditions, pooled and mixed thoroughly. Afterward, the soil was kept in plastic bags at $4^{\circ} \mathrm{C}$ in darkness to keep moisture until the start of the experiment. Immediately before starting the experiment, the soil was cleaned to get rid of clumps and mixed with sand $(0.50 \mathrm{~mm}$ particle size) (Rådasand, Lidköping, Sweden) to a final content of $20 \% \mathrm{vol} / \mathrm{vol}$.

Nematode isolation and characterization. Living nematodes were recovered from $15 \mathrm{~g}$ of soil by using a modified Baermann funnel extraction method (Baermann 1917; Viketoft et al. 2005). Afterward, stereomicroscope (Wild M5A Heerbrugg, Heerbrugg, Switzerland) was used at 50x magnification level to classify each nematode at the level of genera, on the basis of morphological characters.

Fungal culture conditions. $C$. rosea strain IK726 was maintained on potato dextrose agar (PDA, Sigma-Aldrich, Steinheim, Germany). For the pot experiments, $C$. rosea IK726 was formulated as a peat-wheat bran powder formulation modified from Jensen et al. (2000) by using a coffee mill instead of grinding in a mortar. Spore viability in the $C$. rosea IK726 formulation was determined by serial dilutions, where $1 \mathrm{~g}$ of formulation was suspended in $10 \mathrm{ml}$ of water together with one drop of Tween 20 in a sterile 50-ml falcon tube, vortexed vigorously, diluted serially to $10^{-7}$, and plated on PDA plates $(9 \mathrm{~cm}$ diameter). Viable spore concentration was calculated based on colony forming unit (CFU) counts. C. rosea IK726 formulation was mixed with the soil to reach a concentration of $2.5 \times 10^{5}$ viable $\mathrm{CFU} / \mathrm{g}$ of soil.

Climate chamber pot experiment. Plastic pots $(9 \times 9 \times 10 \mathrm{~cm})$ were filled with $430 \mathrm{~g}$ of uninoculated soil or C. rosea-inoculated soil. Seeds of carrot (Daucus carota subsp. sativus 'Bolero' F1) and wheat (Triticum aestivum winter wheat 'Stava') were obtained from Lindbloms Frö (Kivik, Sweden) and Lantmännen Lantbruk (Holmsund, Sweden), respectively. Eight seeds were planted in each pot. Eight pots (biological replicates) per treatment were included in a complete randomized design, and maintained in a climate chamber under controlled conditions: relative humidity $70 \%$, temperature $15^{\circ} \mathrm{C}$, light and darkness 12/12 h. Six treatments were included: (i) soil + carrot plant, (ii) soil + carrot plant $+C$. rosea, (iii) soil + wheat plant, (iv) soil + wheat plant + C. rosea, (v) soil $+C$. rosea, and (vi) soil only. Equal amounts of water were given to each pot throughout the 7-week experimental period.

At the time of harvest, plants were cut at the soil surface and plant shoot fresh weight $(\mathrm{g})$, plant shoot dry weight $(\mathrm{g})$, and plant shoot length $(\mathrm{cm})$ were recorded. Living nematodes were recovered from $15 \mathrm{~g}$ of soil and $1 \mathrm{~g}$ of harvested roots by a modified Baermann funnel extraction method (Baermann 1917; Viketoft et al. 2005) and counted.

Interaction bioassays. For in vitro interaction studies, a mixed nematode community was extracted from the field soil using a modified Baermann funnel method (Baermann 1917; Viketoft et al. 2005) and conidia were obtained from 10-day-old cultures of $C$. rosea maintained on PDA plates. Conidia were dislodged from culture plates by adding sterile water, counted using a hemocytometer (Hausser Scientific, Horsham, PA), and diluted to a concentration of $1 \times 10^{6} \mathrm{conidia} / \mathrm{ml}$.

Four different interaction bioassays were performed. First, C. rosea was inoculated on PDA and solid Czapek Dox (CZ) medium (Sigma-Aldrich, Steinheim, Germany) plates $(9 \mathrm{~cm} \mathrm{di-}$ ameter) and maintained at $25^{\circ} \mathrm{C}$ for 7 days. A $2 \times 2 \mathrm{~cm}$ agar block was then removed from the center of the plate and 80 to 100 nematodes (mixed community) were added to the wells, followed by further incubation at $25^{\circ} \mathrm{C}$ for another 5 days for development of the parasitic process (Gao et al. 1996). The second interaction assay was performed by dropping 200 nematodes onto a 10-day-old PDA culture plate of $C$. rosea. In the third interaction bioassay, $1 \times 10^{6}$ conidia of $C$. rosea were inoculated onto a piece of cellophane membrane and inoculated on PDA plates for 2 days, followed by adding 200 nematodes on the cellophane membrane in order to start the infection experiment (Zou et al. 2010). The fourth interaction bioassay was performed in 24-well plastic plates. Each well contained $1 \mathrm{ml}$ of CZ liquid media that was inoculated with $1 \times 10^{6}$ conidia of $C$. rosea and incubated at $25^{\circ} \mathrm{C}$ for 2 days followed by adding 200 nematodes in each well. Microscope (Leica DM5500 B, Wetzlar, Germany) equipped with a camera (Leica DFC420 C) was used at a varying level of magnifications to evaluate the level of fungal parasitism on nematodes after incubation at $25^{\circ} \mathrm{C}$ in each interaction bioassay for another 2 to 7 days. The experiments were performed in duplicates.

Antibiosis assays. Three antibiosis assays were performed with slightly different experimental conditions. E-flasks containing $30 \mathrm{ml}$ (50 $\mathrm{ml}$ in the second and third experiment) liquid PDB, CZ, malt extract (ME) medium (20 g malt extract per liter of distilled water), modified synthetic nutrient medium (SNA) (Nirenberg 1976) or Vogel's medium (VM) (Vogel 1956) were inoculated with $4 \times 10^{5} C$. rosea conidia/flask $\left(1 \times 10^{6}\right.$ conidia/flask in the second and third experiment) and incubated at $25^{\circ} \mathrm{C}$ on a rotating shaker $(120 \mathrm{rpm})$ for 5 days under dark conditions. Fungal biomass was separated from the broth by filtration, and the culture filtrate was further sterile filtrated through a $0.45 \mathrm{~mm}$ cellulose membrane (Sarstedt, Nümbrecht, Germany). The $\mathrm{pH}$ of the liquid media and culture filtrates was determined with a $744 \mathrm{pH}$ meter (Metrohm Ltd., Herisau, Switzerland). Two milliliters of liquid media or culture filtrate $(800 \mu \mathrm{l}$ in the second and third experiment) and $200 \mu \mathrm{l}$ of water containing 100 nematodes (mixed community) were mixed in wells (24-well plastic plates) and incubated at $25^{\circ} \mathrm{C}$. The numbers of dead and alive nematodes were determined after 24 and $48 \mathrm{~h}$ using a touch assay under a stereomicroscope (Gill et al. 2003) at 50× magnification level. Five biological replicates were included.

In the second experiment, only SNA liquid media and culture filtrates were used. A heat treatment $\left(80^{\circ} \mathrm{C}\right.$ for $\left.1 \mathrm{~h}\right)$ of the culture filtrates was included in order to denature secreted proteins and heat-labile metabolites (Dubey et al. 2014). One hundred nematodes in $200 \mu \mathrm{l}$ of water were added, and the numbers of dead and alive nematodes were determined in five biological replicates after 12, 24, 36, and $48 \mathrm{~h}$.

In the third experiment, PDB liquid media and culture filtrates were used to investigate the specificity of $C$. rosea antibiosis against nematodes. One hundred nematodes in $200 \mu \mathrm{l}$ of water were added, and the numbers of dead and alive nematodes were determined and categorized into genera based on morphological characters in five biological replicates after $24 \mathrm{~h}$ of incubation.

Statistical analyses. All statistical analyses were performed using the open-source statistical software $\mathrm{R}$ (ver. 3.2.5). In the climate chamber pot experiment, the effect of treatments on the number of nematodes was analyzed with Poisson regression where over dispersion, in some cases, was accounted for by using the quasiPoisson distribution. Analyses were made separately with respect to treatments with and without carrot plants and with and without wheat plants. Pairwise comparisons were made using Tukey's method for multiple $P$ value adjustment. Comparisons of average plant weight and length were made using Welch's $t$ test to account for inhomogeneous variances.

In the first antibiosis experiment, the proportions of deceased nematodes were compared between culture and control data separately for the two-time points ( $24 \mathrm{~h}, 48 \mathrm{~h}$ ). A $\chi^{2}$ test was conducted, which is justified considering the high number of counts obtained for each condition and time point. A two-sided test was applied, including Yates' continuity correction. In the second antibiosis 
experiment, comparisons between the three groups (control, culture, heat-treated culture) were made using a $\chi^{2}$ test, employing the null hypothesis of equal proportions of deceased nematodes in all groups. Each time point (12, 24, 36, and $48 \mathrm{~h}$ ) was examined separately. In the third antibiosis experiment, the proportions of deceased nematodes were compared between genera using a $\chi^{2}$ test, employing the null hypothesis of equal proportions of dead nematodes across all genera. Posthoc tests were conducted in order to compare the proportions pairwise. To carry out these tests, the function pairwise proportions test was used. The $P$ values were adjusted for multiple testing by using the Bonferroni approach and false discovery rate (FDR) in the third antibiosis experiment.

\section{RESULTS}

Nematode community composition in infested soil. Initial screening of the sampled soil revealed that it contained a number of plant-parasitic nematode genera. The migratory endoparasitic nematode genus Pratylenchus spp. was the most prevalent, and constituted $38 \%$ of all isolated nematodes (Fig. 1). Sedentary endoparasitic nematodes (Heterodera spp.), ectoparasitic nematodes (Tylenchorhynchus spp., Paratylenchus spp. and Trichodorus spp.) constituted 3 to $6 \%$ of the community, while migratory ecto- or semiendoparasitic nematodes (Helicotylenchus spp. and Rotylenchus spp.) made up 5 and $3 \%$ of the community, respectively. Nonparasitic nematodes constituted $36 \%$ of the community (bacterivores $13 \%$, fungivores $17 \%$, and omnivores 6\%) as shown in Figure 1. Because of the dominance of Pratylenchus spp. in the soil, carrot, and wheat were chosen as experimental crops, as these crops are reported to be suitable hosts for Pratylenchus spp. (Moens and Perry 2009).

Effect of $C$. rosea on nematode communities in soil and plant roots. All plant-parasitic nematodes decreased in numbers in soil from pots with growing plants (carrot as well as wheat) when C. rosea was applied $(P \leq 0.005)$, with the exception of Paratylenchus in carrot and Trichodorus in wheat (Figs. 2 and 3). Interestingly, nonparasitic nematodes (bacterivores, fungivores and omnivores) were not affected by the $C$. rosea treatment, neither in carrot nor wheat (Figs. 2 and 3). The addition of $C$. rosea into soil reduced the number of Pratylenchus and Heterodera endoparasitic nematodes extracted from roots of both carrot $(P \leq 0.001)$ and wheat $(P \leq 0.01)$ compared with the control treatment without $C$. rosea (Fig. 4).

In soil without plants, the numbers of Pratylenchus, Heterodera (J2), Helicotylenchus, and Trichodorus nematodes decreased $(P \leq$ 0.05 ) in the $C$. rosea treatment compared with soil without $C$. rosea (Figs. 2 and 3).
Plant growth. Control plants, but not $C$. rosea-treated plants, displayed yellowing of leaves and chlorosis (data not shown). Mixing in $C$. rosea in the soil increased fresh shoot weight $(P=$ $0.019)$ and shoot length $(P<0.001)$ of wheat, while only shoot dry weight $(P=0.036)$ increased in carrot (Table 1$)$.

Interaction bioassays. Microscopic investigations of all four tested interaction bioassays failed to reveal any indication of fungal attachment or penetration, as signs of direct parasitism of $C$. rosea to nematodes (data not shown).

Antibiosis assays. Culture filtrates from $C$. rosea grown in PDB, ME, and SNA media all showed varying levels of toxicity to nematodes, compared with the media only control treatment, after both 24 and $48 \mathrm{~h}$ (Fig. 5). The highest toxicity was detected in the SNA culture filtrate where $100 \%$ of the nematodes were killed already after $24 \mathrm{~h}$ of incubation, while only $36 \%$ were killed in the corresponding medium control. Neither VM nor CZ media were suitable for nematode survival as even the media control treatments (without $C$. rosea growth) resulted in more than $96 \%$ dead nematodes already after $24 \mathrm{~h}$ (Fig. 5).

Due to the high nematicidal toxicity of SNA $C$. rosea culture filtrates, this medium was chosen to evaluate if the nematicidal activity was heat-stable. The heat treatment decreased, but did not abolish, the toxicity toward nematodes in the $C$. rosea culture filtrates after $12 \mathrm{~h}$ of incubation (Fig. 6). Prolonged incubation time revealed no difference in nematode mortality between the heattreated culture filtrates and the medium controls (Fig. 6).

There were no significant differences $(P \geq 0.085)$ in mortality between nematode genera or groups incubated in PDB. In contrast, all plant-parasitic nematode genera exhibited higher mortality in culture filtrates from $C$. rosea grown in PDB $(P \leq 0.001)$ compared with nonparasitic nematode groups (Table 2$)$.

\section{DISCUSSION}

The proportions of plant-parasitic nematode genera found in the soil used for the experiment, including the dominance of Pratylenchus spp., is in agreement with a previous evaluation of the nematode community in soil from the same field (Schmidt et al. 2017). The dominating species were identified as $P$. crenatus and $P$. neglectus (Schmidt et al. 2017).

Soil application of $C$. rosea lowered the populations of all studied plant-parasitic nematode genera in soil under the influence of both carrot and wheat, with the exception for Trichodorus under wheat and Paratylenchus under carrot. This reduction in plant-parasitic nematode numbers also correlated with better growth performance

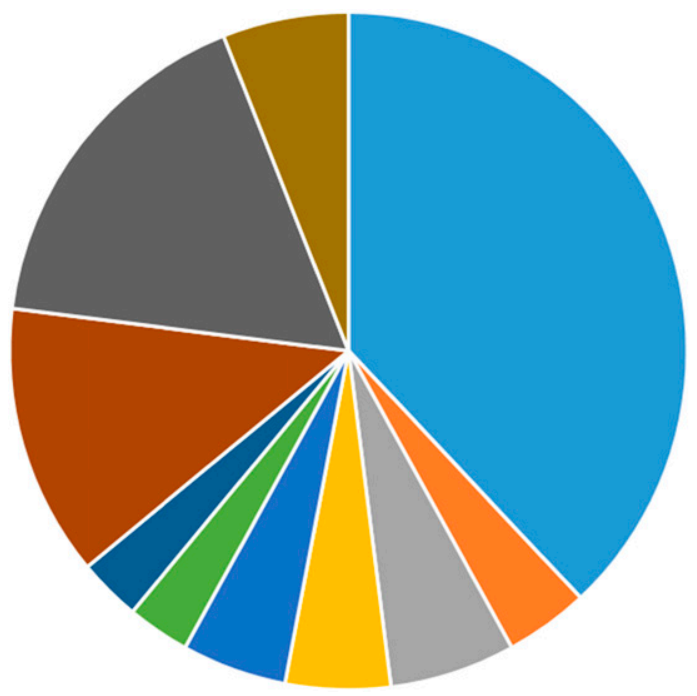

\footnotetext{
- Pratylenchus (38\%)

- Heterodera (J2- $4 \%)$

- Tylenchorhynchus $(6 \%)$

- Paratylenchus (5\%)

- Helicotylenchus (5\%)

- Rotylenchus (3\%)

- Trichodorous (3\%)

- Bacterivores (13\%)

- Fungivores (17\%)

- Omnivores (6\%)
}

Fig. 1. Percentages of plant-parasitic nematode genera and trophic groups recovered from naturally nematode infested soil, used in pot experiments, interaction bioassays, and antibiosis assays. 


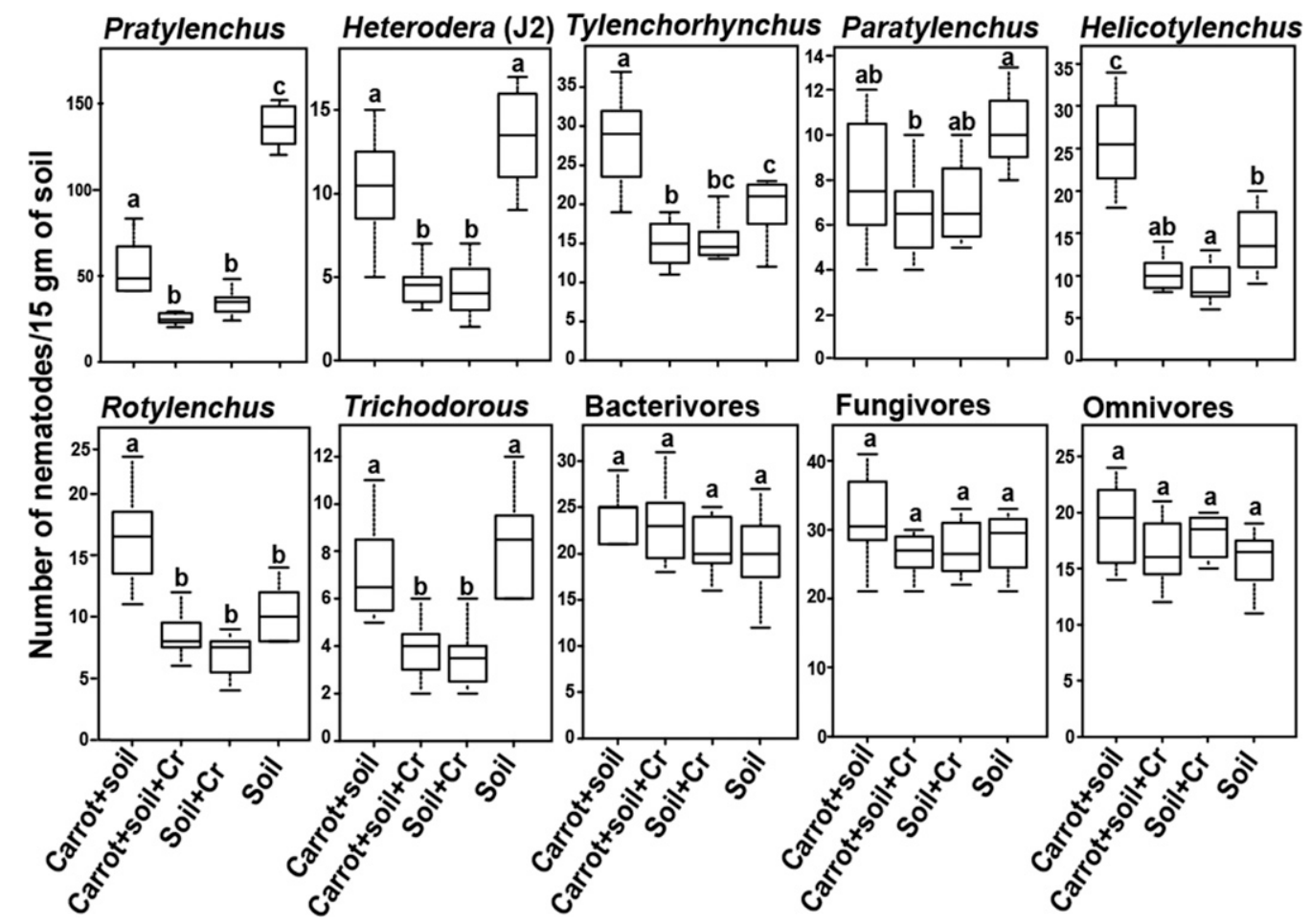

Fig. 2. Effect of Clonostachys rosea IK726 on plant-parasitic nematode genera and trophic groups recovered from carrot plant soil. Horizontal axis shows the different treatment combinations in which "Cr" stands for $C$. rosea while the vertical axis represents the number of nematodes extracted per $15 \mathrm{~g}$ of soil. Each panel represents different genera or groups of nematodes. Treatments marked with different letters for each nematode group are significantly different as determined by the Tukey method $(P<0.05)$. Eight biological replications were included per treatment. Bars are shown medians and boxes the 25th and 75 th percentiles.

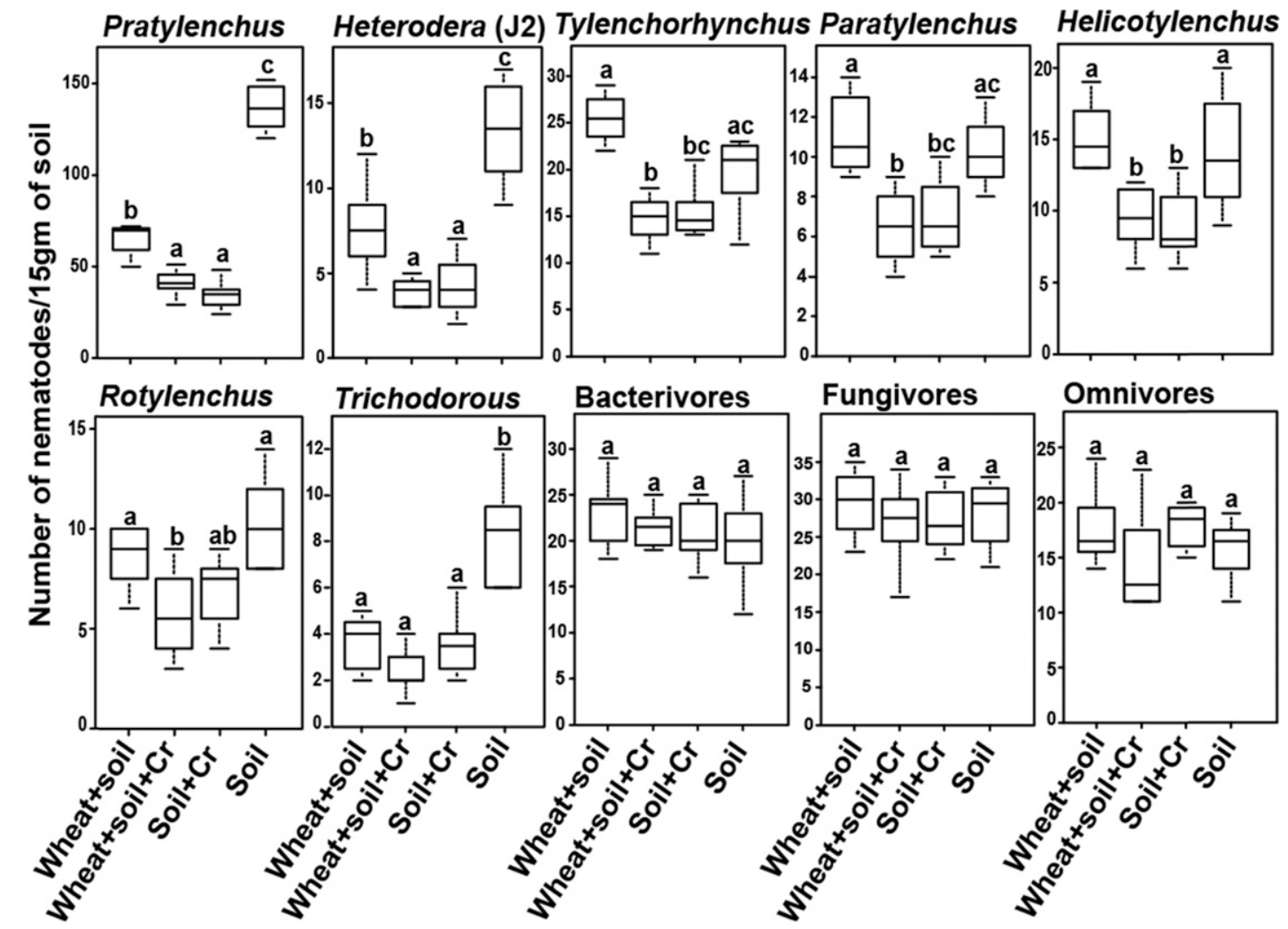

Fig. 3. Effect of Clonostachys rosea IK726 on plant-parasitic nematode genera and trophic groups recovered from wheat plant soil. Horizontal axis shows the different treatment combinations in which "Cr" stands for $C$. rosea while the vertical axis represents the number of nematodes extracted per $15 \mathrm{~g}$ of soil. Each panel shows a particular genus or group of nematodes. Treatments marked with different letters for each nematode group are significantly different as determined by the Tukey method $(P<0.05)$. Note that data for the Soil and Soil $+\mathrm{Cr}$ treatments are the same as in Figure 1 but included here for clarity. Eight biological replications were included per treatment. Bars represent medians and boxes the 25 th and 75 th percentiles. 
of both carrot and wheat plants, and lack of disease symptoms (yellowing of leaves and chlorosis) typically associated with nematode damage of roots (Jones et al. 2013). This shows that C. rosea strain IK 726 can control nematode disease in these systems, and it is to our knowledge the first time a biocontrol effect on plantparasitic nematodes is reported for $C$. rosea. Nematode in vitro antagonism, but not biological control, of another strain of $C$. rosea was previously reported ( $\mathrm{Li}$ et al. 2006; Zou et al. 2010). C. rosea was also able to lower the populations of Pratylenchus spp., Heterodera spp., Helicotylenchus spp., and Trichodorus spp. in soil alone, without plants. This suggests that $C$. rosea has a direct effect on nematode survival that is not dependent on the presence of plants.

Other beneficial fungi reported to control plant-parasitic nematodes include Trichoderma koningii and T. harzianum against the root knot nematode Meloidogyne arenaria (Sharon et al. 2001; Windham et al. 1989), and T. harzianum against the citrus nematode
Tylenchulus semipenetrans (Reddy et al. 1996). More recently, Zhou et al. (2016) reported that an endophytic isolate of Phialemonium inflatum (previously Paecilomyces inflatus) can restrain penetration, galling, and subsequent reproduction of Meloidogyne incognita in cotton plants. The egg pathogenic fungus Purpureocillium lilacinum (previously Paecilomyces lilacinus) is extensively tested against plantparasitic nematodes (Atkins et al. 2005) and it successfully control $M$. javanica and the potato cyst nematode Globodera rostochiensis (Cannayane and Sivakumar 2001). However, its biological control potential is inconsistent under field conditions (Kerry and Evans 1996). P. lilacinum and the nematode-trapping fungus Monacrosporium lysipagum, either alone or in combination, are able to reduce $M$. javanica galls and juveniles by 62 and $94 \%$, respectively, and to reduce cysts caused by $H$. avenae by $65 \%$ (Khan et al. 2006). Conidia produced by the nematode endoparasitic fungus Hirsutella minnesotensis adhere to and penetrate the cuticle of second stage

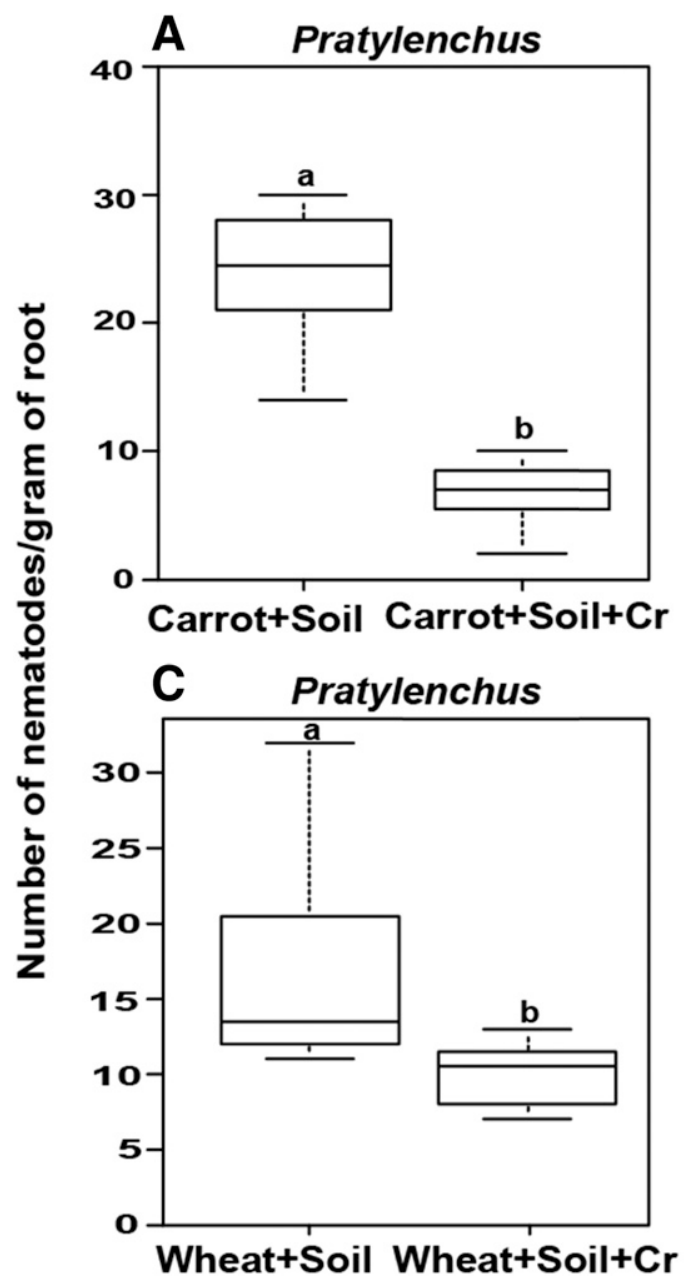

B
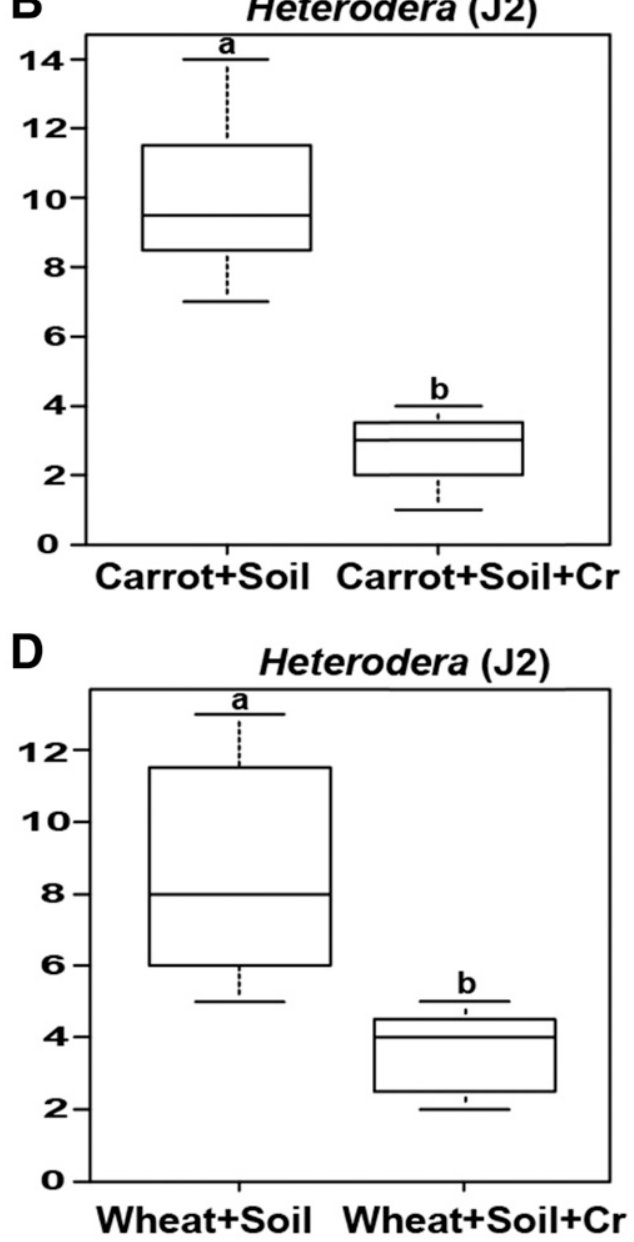

Fig. 4. Effect of Clonostachys rosea IK726 on Pratylenchus and Heterodera (J2) nematodes recovered from plant roots. Horizontal axis shows the different treatment combinations in which "Cr" stands for C. rosea while the vertical axis represents the number of nematodes extracted per 1 gram of roots. A and $\mathbf{B}$ represents nematodes species recovered from carrot plant roots, while $\mathbf{C}$ and $\mathbf{D}$ represents nematodes species recovered from wheat plant roots. Treatments marked with different letters within each panel are significantly different as determined by the Tukey method $(P<0.05)$. Eight biological replications were included per treatment. Bars represent medians and boxes the 25 th and 75 th percentiles.

TABLE 1. Effect of soil application of Clonostachys rosea IK726 on shoot weight and shoot length of carrot and wheat ${ }^{2}$

\begin{tabular}{lcccr}
\hline Plant & C. rosea & Fresh shoot weight $(\mathrm{g})$ & Dry shoot weight $(\mathrm{g})$ & Shoot length $(\mathrm{cm})$ \\
\hline Carrot & - & $0.16 \pm 0.02 \mathrm{a}$ & $0.03 \pm 0.00 \mathrm{a}$ & $9.03 \pm 0.36 \mathrm{a}$ \\
Carrot & + & $0.24 \pm 0.10 \mathrm{a}$ & $0.04 \pm 0.02 \mathrm{~b}$ & $10.03 \pm 1.73 \mathrm{a}$ \\
Wheat & - & $0.15 \pm 0.02 \mathrm{a}$ & $0.04 \pm 0.00 \mathrm{a}$ & $16.86 \pm 1.23 \mathrm{a}$ \\
Wheat & + & $0.18 \pm 0.02 \mathrm{~b}$ & $0.04 \pm 0.00 \mathrm{a}$ & $20.94 \pm 1.77 \mathrm{~b}$ \\
\hline
\end{tabular}

${ }^{\mathrm{z}}$ Average shoot weight and length ( \pm standard deviation) is given per plant. Different letters $(\mathrm{a}$ and $\mathrm{b})$ indicate statistically significant differences $(P \leq 0.05)$ within carrot or wheat, respectively, using Student's $t$ test. 


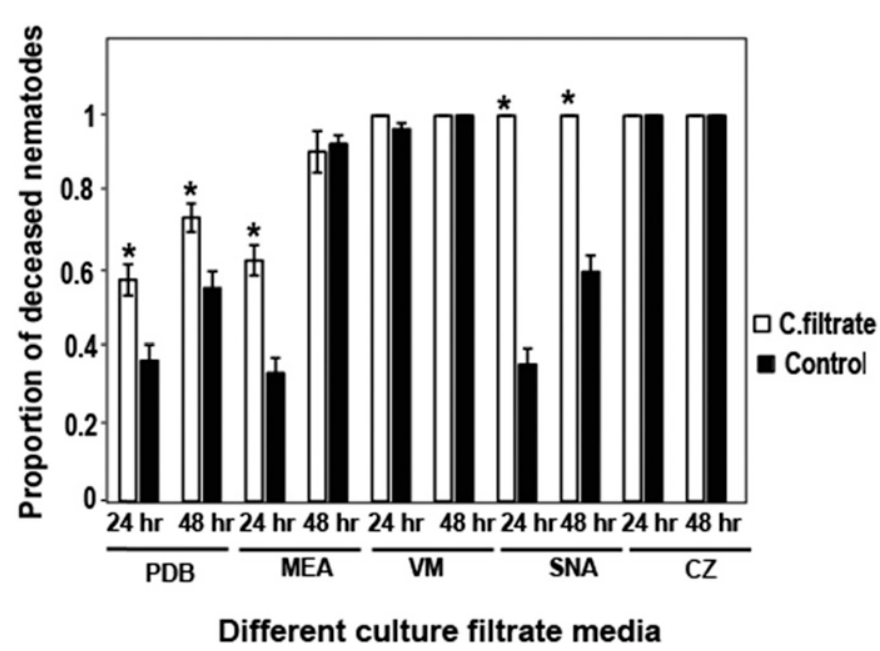

Fig. 5. Nematode mortality in different Clonostachys rosea culture filtrates. Nematode mortality was assessed in C. rosea culture filtrates and compared with the corresponding medium control, after 24 and $48 \mathrm{~h}$ of incubation. The $95 \%$ confidence intervals for the proportions based on five biological replicates are shown as error bars. Culture filtrates marked with an asterisk are significantly $(P \leq 0.05)$ different from the corresponding control as determined by the $\chi^{2}$ test. $\mathrm{PDB}=$ potato dextrose broth, $\mathrm{ME}=$ malt extract medium, $\mathrm{VM}=$ Vogel's medium, $\mathrm{SNA}=$ modified synthetic nutrient medium, $\mathrm{CZ}=$ Czapek dox medium.

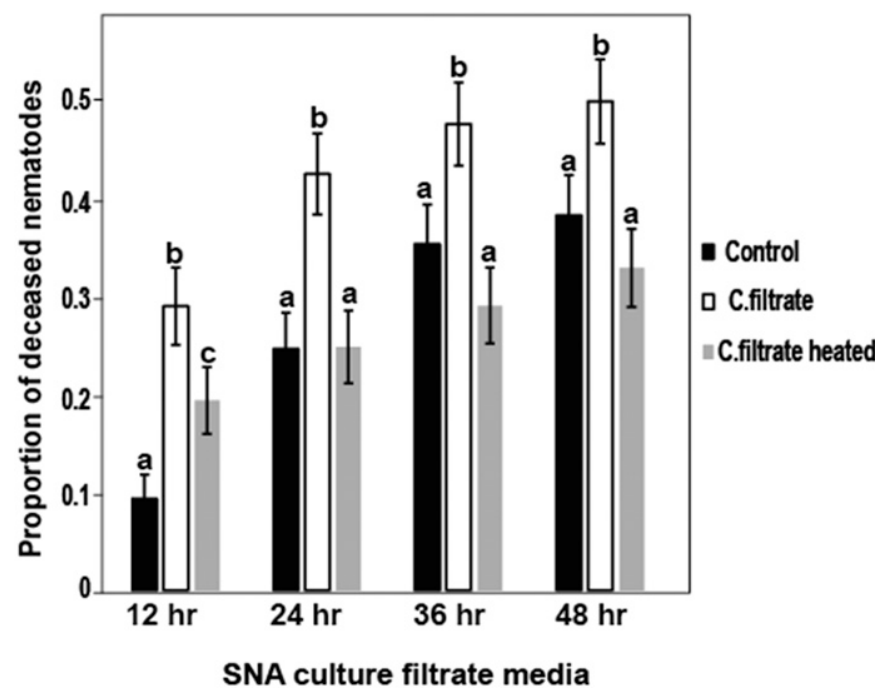

Fig. 6. Nematode mortality in modified synthetic nutrient medium Clonostachys rosea culture filtrates over time. Nematode mortality was assessed in C. rosea modified synthetic nutrient medium culture filtrates, heat-treated culture filtrates and in the corresponding medium control, after 12, 24, 36, and $48 \mathrm{~h}$ of incubation. The $95 \%$ confidence intervals for the proportions based on five biological replicates are shown as error bars. Treatments marked with different letters within each time point are significantly $(P \leq 0.05)$ different as determined by the $\chi^{2}$ test. juveniles of cyst nematodes, ultimately killing the nematode (Lai et al. 2014). The nematode-trapping fungi Arthrobotrys oligospora (adhesive nets) and $A$. dactyloides (constricting rings) form specific hyphal traps used to trap and kill the sugar beet cyst nematode $H$. schachtii and the northern root knot nematode $M$. hapla, respectively (Andersson et al. 2014).

The numbers of Pratylenchus spp. nematodes decreased in soil grown with both carrot and wheat seedlings compared with in soil alone, but based on the migratory endoparasitic lifestyle and high numbers of Pratylenchus nematodes recovered from carrot and wheat roots this is not surprising. It appears that Pratylenchus nematodes migrated from the soil into the plant roots during the experiment, and a similar pattern was also observed for Heterodera spp. where the second stage juveniles also migrated from soil into plant roots.

For Tylenchorhynchus and Rotylenchus in both carrot and wheat, and for Paratylenchus in wheat, $C$. rosea was only able to control the plant-parasitic nematode numbers in the presence of plant roots. This may suggest the involvement of induced local resistance triggered by $C$. rosea, which is previously suggested to occur in wheat and tomato (Mouekouba et al. 2014; Roberti et al. 2008). An alternative explanation is that the lack of root exudates has a negative effect on the activity of $C$. rosea. It has been shown previously by using a $g f p$ transformant of $C$. rosea IK726 that it is germinating and actively growing in soil in the beginning when applied as a wheat bran formulation exploiting the wheat bran as a carbon source. It was, however, observed that after a short time the fungus was present mainly as conidia in bulk soil following application as a wheat bran formulation (Lübeck et al. 2002). Our findings are in accordance with the results discussed by Lübeck et al. (2002) indicating the importance of root exudates for the activity of $C$. rosea.

The data regarding plant-parasitic nematode populations show a certain level of specificity in the activity of $C$. rosea, something that is further evident by the finding that the populations of bacterivores, fungivores, and omnivores nematodes are not lowered by soil application of $C$. rosea. In addition, this is further supported by the result of our antibiosis assay where plant-parasitic nematodes, but not nonparasitic nematodes, were affected by secreted nematicidal metabolites or enzymes from $C$. rosea. Similar results were reported by Hallmann and Sikora (1996) with culture filtrates of Fusarium oxysporum. In their study, plant-parasitic nematode species were inactivated by 60 to $100 \%$ whereas bacterivores and fungivores species were not affected. Our findings are also in agreement with a study by Cayrol et al. (1989) where culture filtrates of $P$. lilacinum inactivated up to $100 \%$ of plant-parasitic nematodes, while fungivores, bacterivores and entomopathogenic species were inactivated to an average of $25 \%$ only. C. rosea strain IK726 is reported to effectively colonize the root surface of tomato (Karlsson et al. 2015), and it may therefore have a higher impact on plantparasitic nematodes that live in the same ecological niche (Hallmann and Sikora 1996). Nematicidal compounds produced by $C$. rosea may also display specificity in action; Dong et al. (2005) reported that dimeric epipolysulfanyldioxopiperazines produced by $C$. rosea strain $1 \mathrm{~A}$ were toxic to the nematodes Caenorhabditis elegans

TABLE 2. Effect of Clonostachys rosea IK726 potato dextrose broth culture filtrates on mortality of plant-parasitic and nonparasitic nematode genera ${ }^{\mathrm{z}}$

\begin{tabular}{|c|c|c|c|c|c|c|c|c|c|c|}
\hline \multirow[b]{2}{*}{ Treatment } & \multicolumn{10}{|c|}{ Nematode genera } \\
\hline & Pratylenchus & Heterodera & Tylenchorhynchus & Paratylenchus & Helicotylenchus & Rotylenchus & Trichodorus & Bacterivores & Fungivores & Omnivores \\
\hline $\begin{array}{l}\text { Culture } \\
\text { filtrate }\end{array}$ & $80.56 \pm 2.95 \mathrm{a}$ & $84.64 \pm 5.77 \mathrm{a}$ & $78.57 \pm 7.75 \mathrm{a}$ & $65.52 \pm 8.82 \mathrm{a}$ & $80.00 \pm 5.39 \mathrm{a}$ & $82.61 \pm 7.90 \mathrm{a}$ & $64.00 \pm 9.60 \mathrm{a}$ & $11.59 \pm 3.85 b$ & $12.79 \pm 3.60 \mathrm{~b}$ & $8.89 \pm 4.24 b$ \\
\hline $\begin{array}{l}\text { Medium } \\
\text { control }\end{array}$ & $21.08 \pm 3.16 \mathrm{a}$ & $25.64 \pm 6.99 a$ & $24.14 \pm 7.94 \mathrm{a}$ & $23.33 \pm 7.72 \mathrm{a}$ & $26.92 \pm 6.15 \mathrm{a}$ & $27.27 \pm 9.49 \mathrm{a}$ & $20.83 \pm 8.29 a$ & $9.59 \pm 3.44 \mathrm{a}$ & $10.34 \pm 3.26 \mathrm{a}$ & $13.79 \pm 4.52 \mathrm{a}$ \\
\hline
\end{tabular}


and Panagrellus redivivus but not to the pine wood nematode Bursaphelenchus xylophilus.

The observation that $C$. rosea strain IK726 can successfully control plant-parasitic nematodes in the soil, even without the presence of a plant, indicate that direct parasitism or antibiosis is at least partially involved in the interaction. Despite several attempts using different interaction systems, we failed to demonstrate any evidence of direct parasitism of $C$. rosea strain IK726 on nematodes. This is in contrast to $C$. rosea strain 611 that is reported to parasitize the bacterial feeding nematode $P$. redivivus $(\mathrm{Li}$ et al. 2006; Zou et al. 2010). Therefore, we conclude that the ability to parasitize nematodes may differ between different strains of C. rosea. Instead, culture filtrates from C. rosea IK726 displayed varying levels of toxicity toward a mixed community of nematodes, indicating production and secretion of nematicidal metabolites and enzymes. The previously mentioned nematicidal epipolysulfanyldioxopiperazines was produced by $C$. rosea strain 1A grown on wheat kernels for 20 days (Dong et al. 2005), and it is likely that similar compounds are produced by $C$. rosea strain IK726 in the liquid culture conditions used in the present study. C. rosea strain 611 is also shown to produce the extracellular serine protease $\mathrm{PrC}$ (Li et al. 2006) during growth on PDB, which killed $80 \%$ of $P$. redivivus nematodes after $48 \mathrm{~h}$ of incubation. Interestingly, the crude extract displayed even higher toxicity, despite lower protease activity, which suggests the presence of additional nematicidal compounds or enzymes. Boiling of the $C$. rosea strain 611 crude extracts abolished up to $80 \%$ of the nematicidal activity, very similar to the results for the $C$. rosea strain IK726 in the current work. This shows that the major nematicidal activity in culture filtrates of $C$. rosea stems from heat-labile compounds or enzymes, but a minor part of the activity remains after heat treatment.

This study demonstrates that $C$. rosea strain IK726 can decrease the populations of several plant-parasitic nematodes in naturally nematode infested soil, and thereby improve growth of carrot and wheat. Antibiosis through secreted metabolites or enzymes contributes to the antagonistic effect, while direct parasitism of nematodes was not detected.

\section{ACKNOWLEDGMENTS}

We thank the Swedish Research Council for Environment, Agricultural Sciences and Spatial Planning (FORMAS) (grant number 220-2014-289) and the Plant Protection Platform at the Swedish University of Agricultural Sciences for financial support to this study. S. Håkansson is acknowledged for providing the $C$. rosea formulation.

\section{LITERATURE CITED}

Andersson, K. M., Kumar, D., Bentzer, J., Friman, E., Ahrén, D., and Tunlid, A. 2014. Interspecific and host-related gene expression patterns in nematodetrapping fungi. BMC Genomics 15:968.

Atkins, S. D., Clark, I. M., Pande, S., Hirsch, P. R., and Kerry, B. R. 2005. The use of real-time PCR and species-specific primers for the identification and monitoring of Paecilomyces lilacinus. FEMS Microbiol. Ecol. 51:257-264.

Baermann, G. 1917. Eine einfache methode zur auffindung von ankylostomum (Nematoden) larven in erdproben. Geneeskunding Tijdschr. NederlandschIndie 57:131-137.

Barker, K. R., and Koenning, S. R. 1998. Developing sustainable systems for nematode management. Annu. Rev. Phytopathol. 36:165-205.

Beketov, M. A., Kefford, B. J., Schäfer, R. B., and Liess, M. 2013. Pesticides reduce regional biodiversity of stream invertebrates. Proc. Natl. Acad. Sci. 110:11039-11043.

Cannayane, I., and Sivakumar, C. 2001. Nematode egg-parasitic fungus I: Paecilomyces lilacinus-A review. Agric. Rev. 22:79-86.

Cayrol, J.-C., Djian, C., and Pijarowski, L. 1989. Study of the nematicidal properties of the culture filtrate of the nematophagous fungus Paecilomyces lilacinus. Rev. Nematol. 12:331-336.

Decraemer, W., and Hunt, D. J. 2013. Structure and classification. Pages 3-39 in: Plant Nematology, 2nd ed. R. N. Perry and M. Moens, eds. CABI Publisher, Wallingford, England.
Dong, J. Y., He, H. P., Shen, Y. M., and Zhang, K. Q. 2005. Nematicidal epipolysulfanyldioxopiperazines from Gliocladium roseum. J. Nat. Prod. 68:1510-1513.

Dubey, M. K., Jensen, D. F., and Karlsson, M. 2014. An ATP-binding cassette pleiotropic drug transporter protein is required for xenobiotic tolerance and antagonism in the fungal biocontrol agent Clonostachys rosea. Mol. PlantMicrobe Interact. 27:725-732.

Gan, Z., Yang, J., Tao, N., Yu, Z., and Zhang, K. 2007. Cloning and expression analysis of a chitinase gene Crchil from the mycoparasitic fungus Clonostachys rosea (syn. Gliocladium roseum). J. Microbiol. 45:422-430.

Ganguly, S., and Ganguly, A. 2008. Pratylenchus (Nematoda: Pratylenchidae): Diagnosis, biology, pathogenicity and management. Indian J. Nematol. 38:126.

Gao, R., Lei, L., and Liu, X. 1996. A simple method for inducing and observing predacious devices of nematode trapping fungi. Acta Mycol. Sin. 15:304-305.

Gill, M. S., Olsen, A., Sampayo, J. N., and Lithgow, G. J. 2003. An automated high-throughput assay for survival of the nematode Caenorhabditis elegans. Free Radic. Biol. Med. 35:558-565.

Hallmann, J., and Sikora, R. 1996. Toxicity of fungal endophyte secondary metabolites to plant parasitic nematodes and soil-borne plant pathogenic fungi. Eur. J. Plant Pathol. 102:155-162.

Jensen, B., Knudsen, I. M., and Jensen, D. F. 2000. Biological seed treatment of cereals with fresh and long-term stored formulations of Clonostachys rosea: Biocontrol efficacy against Fusarium culmorum. Eur. J. Plant Pathol. 106:233-242.

Jensen, D. F., Knudsen, I. M., Mamarabadi, M., Hockenhull, J., and Jensen, B. 2007. Development of a biocontrol agent for plant disease control with special emphasis on the near commercial fungal antagonist Clonostachys rosea strain 'IK726'. Australas. Plant Pathol. 36:95-101.

Jones, J. T., Haegeman, A., Danchin, E. G., Gaur, H. S., Helder, J., Jones, M. G., Kikuchi, T., Manzanilla López, R., Palomares Rius, J. E., Wesemael, W. M., and Perry, R. N. 2013. Top 10 plant-parasitic nematodes in molecular plant pathology. Mol. Plant Pathol. 14:946-961.

Karlsson, M., Durling, M. B., Choi, J., Kosawang, C., Lackner, G., Tzelepis, G. D., Nygren, K., Dubey, M. K., Kamou, N., Levasseur, A., Zapparata, A., Wang, J., Amby, D. B., Jensen, B., Sarrocco, S., Panteris, E., Lagopodi, A. L., Pöggeler, S., Vannacci, G., Collinge, D. B., Hoffmeister, D., Henrissat, B., Lee, Y. H., and Jensen, D. F. 2015. Insights on the evolution of mycoparasitism from the genome of Clonostachys rosea. Genome Biol. Evol. 7:465-480.

Kerry, B. R., and Evans, K. 1996. New strategies for the management of plant parasitic nematodes. Pages 134-152 in: Principles and Practice of Managing Soil Borne Plant Pathogens. R. Hall, ed. American Phytopathological Society, St. Paul, MN.

Khan, A., Williams, K. L., and Nevalainen, H. K. 2006. Control of plantparasitic nematodes by Paecilomyces lilacinus and Monacrosporium lysipagum in pot trials. BioControl 51:643-658.

Lai, Y., Liu, K., Zhang, X., Zhang, X., Li, K., Wang, N., Shu, C., Wu, Y., Wang, C., and Bushley, K. E. 2014. Comparative genomics and transcriptomics analyses reveal divergent lifestyle features of nematode endoparasitic fungus Hirsutella minnesotensis. Genome Biol. Evol. 6: 3077-3093

Li, J., Yang, J., Huang, X., and Zhang, K. Q. 2006. Purification and characterization of an extracellular serine protease from Clonostachys rosea and its potential as a pathogenic factor. Process Biochem. 41:925-929.

Lübeck, M., Knudsen, I. M., Jensen, B., Thrane, U., Janvier, C., and Jensen, D. F. 2002. GUS and GFP transformation of the biocontrol strain Clonostachys rosea IK726 and the use of these marker genes in ecological studies. Mycol. Res. 106:815-826.

Milligan, S. B., Bodeau, J., Yaghoobi, J., Kaloshian, I., Zabel, P., and Williamson, V. M. 1998. The root knot nematode resistance gene $M i$ from tomato is a member of the leucine zipper, nucleotide binding, leucine-rich repeat family of plant genes. Plant Cell 10:1307-1319.

Moens, M., and Perry, R. N. 2009. Migratory plant endoparasitic nematodes: A group rich in contrasts and divergence. Annu. Rev. Phytopathol. 47:313-332.

Mouekouba, L. D. O., Zhang, L., Guan, X., Chen, X., Chen, H., Zhang, J., Zhang, J., Li, J., Yang, Y., and Wang, A. 2014. Analysis of Clonostachys rosea-induced resistance to tomato gray mold disease in tomato leaves. PLoS One 9:e102690.

Nicol, J. M., Turner, S. J., Coyne, D., Den Nijs, L., Hockland, S., and Maafi, Z. T. 2011. Current nematode threats to world agriculture. Pages 21-43 in: Genomics and Molecular Genetics of Plant-Nematode Interactions. J. Jones, G. Gheysen, and C. Fenoll, eds. Springer, Dordrecht, The Netherlands.

Nirenberg, H. 1976. Untersuchungen uber die morphologische und biologische differenzierung in der Fusarium-sektion Liseola. Mitt. Biol. Bundesanst. Land-u. Forstw. 169:1-117.

Reddy, P. P., Rao, M., and Nagesh, M. 1996. Management of citrus nematode, Tylenchulus semipenetrans, by integration of Trichoderma harzianum with oil cakes. Nematol. Mediterr. 24:265-267. 
Roberti, R., Veronesi, A., Cesari, A., Cascone, A., Di Berardino, I., Bertini, L., and Caruso, C. 2008. Induction of PR proteins and resistance by the biocontrol agent Clonostachys rosea in wheat plants infected with Fusarium culmorum. Plant Sci. 175:339-347.

Schmidt, J. H., Bergkvist, G., Campiglia, E., Radicetti, E., Wittwer, R. A., Finckh, M. R., and Hallmann, J. 2017. Effect of tillage, subsidiary crops, and fertilization on plant-parasitic nematodes in a range of agro-environmental conditions within Europe. Ann. Appl. Biol. 171:477-489.

Schroers, H. J., Samuels, G. J., Seifert, K. A., and Gams, W. 1999. Classification of the mycoparasite Gliocladium roseum in Clonostachys as C. rosea, its relationship to Bionectria ochroleuca, and notes on other Gliocladium-like fungi. Mycologia 91:365-385.

Sharon, E., Bar-Eyal, M., Chet, I., Herrera-Estrella, A., Kleifeld, O., and Spiegel, Y. 2001. Biological control of the root-knot nematode Meloidogyne javanica by Trichoderma harzianum. Phytopathology 91:687-693.

Tzelepis, G., Dubey, M., Jensen, D. F., and Karlsson, M. 2015. Identifying glycoside hydrolase family 18 genes in the mycoparasitic fungal species Clonostachys rosea. Microbiology 161:1407-1419.

Viglierchio, D. R., and Brown, S. M. 1989. In vitro testing for nonfumigantnematicide resistance in Heterodera schachtii. Rev. Nematol. 12:139-143.
Viketoft, M., Palmborg, C., Sohlenius, B., Huss Danell, K., and Bengtsson, J. 2005. Plant species effects on soil nematode communities in experimental grasslands. Appl. Soil Ecol. 30:90-103.

Vogel, H. 1956. A convenient growth medium for Neurospora (Medium N). Microbiol. Genet. Bull. 13:42-43.

Williamson, V. M., and Gleason, C. A. 2003. Plant-nematode interactions. Curr. Opin. Plant Biol. 6:327-333.

Windham, G., Windham, M., and Williams, W. 1989. Effects of Trichoderma spp. on maize growth and Meloidogyne arenaria reproduction. Plant Dis. 73:493-495.

Yamashita, T. T., Viglierchio, D. R., and Schmitt, R. V. 1986. Responses of nematodes to nematicidal applications following extended exposures to subnematicidal stress. Rev. Nematol. 9:49-60.

Zhou, W., Wheeler, T. A., Starr, J. L., Valencia, C. U., and Sword, G. A. 2016. A fungal endophyte defensive symbiosis affects plant-nematode interactions in cotton. Plant Soil. doi.org/10.1007/s11104-016-3147-z

Zou, C. G., Tao, N., Liu, W. J., Yang, J. K., Huang, X. W., Liu, X. Y., Tu, H. H., Gan, Z. W., and Zhang, K. Q. 2010. Regulation of subtilisin-like protease $\operatorname{prC}$ expression by nematode cuticle in the nematophagous fungus Clonostachys rosea. Environ. Microbiol. 12:3243-3252. 